\title{
Basic Research - Technology
}

\section{Timing for Composite Resin Placement on Mineral Trioxide Aggregate}

Masaki Tsujimoto, DDS, PhD, ${ }^{* \|}$ Yasuhisa Tsujimoto, DDS, PhD, ${ }^{* *}$ Atsushi Ookubo, DDS, PhD, "Takanobu Shiraishi, M.Eng., PhD, ${ }^{* * *}$ Ikuya Watanabe, DDS, PhD, ${ }^{* * *}$ Shizuka Yamada, DDS, PhD, ${ }^{*}$ Yoshihiko Hayashi, DDS, PhD

From *Department of Cariology, Nagasaki University Graduate School of Biomedical Sciences, Nagasaki, Japan, "Hiu Dental Clinic Institute, Sasebo, Japan, $\quad{ }^{* *}$ Department of Endodontics, Nihon University School of Dentistry at Matsudo, Matsudo, Japan, ${ }^{* * *}$ Department of Dental and Biomedical Materials Science, Nagasaki University Graduate School of Biomedical Sciences, Nagasaki, Japan

Address requests for reprints to Dr. Yoshihiko Hayashi, Department of Cariology, Nagasaki University Graduate School of Biomedical Sciences, Sakamoto 1-7-1, Nagasaki 852-8588, Japan.

E-mail address: hayashi@nagasaki-u.ac.jp

Acknowledgments

The authors deny any conflicts of interest related to this study. 


\begin{abstract}
Introduction: The aim of this study was to investigate the proper timing on restoring composite resin over mineral trioxide aggregate (MTA). Methods: Thirty-five samples of MTA blocks were divided into seven groups with three different times (10 minutes, 1 day, and 7 days) selected for restoring the composite resin over MTA with and without bonding resin, and a control group was included for comparison purposes. After 21 days, the distances between the MTA and composite resin, or between the MTA and bonding agent on sectioned planes along the long axis were measured using a scanning electron microscope (magnification: $\times 2,000$ ). The hardness of MTA near the composite resin was presented as the Vickers microhardness. Results: There were no gaps at the interface in the 10-minute groups, the 1-day group with bonding agent, and the 7-day group with bonding agent. The groups without bonding agent at 1 and 7 days presented a separation or gap at the interface. The value of the Vickers microhardness in the 1-day groups significantly decreased compared to the other groups, regardless of the presence or absence of a bonding agent. Conclusions: These findings suggest that composite resin with a bonding agent over MTA can be restored almost immediately after MTA mixing as a single visit procedure.
\end{abstract}

\title{
Key Words
}

Mineral trioxide aggregate, composite resin, moist curing, scanning electron microscope, Vickers microhardness 
Mineral trioxide aggregate (MTA) is composed of a hydrophilic powder, which reacts with water and sets through the process of hydration (1). MTA has been widely used as root-end and root canal filling materials (2-7), a root or furcal perforation repair material $(8,9)$, a pulp-capping material $(10-14)$, apexification $(15,16)$, and pulpotomy $(17,18)$. Although MTA is now thought to be an indispensable biomaterial in the endodontic fields, the main disadvantage is its prolonged setting time (initial: after $24 \mathrm{~h}$ to $40 \mathrm{MPa}$ of compressive strength (CS) and 45 minutes; final: after 21 days to $67 \mathrm{MPa}$ of CS) (19).

This study is the first report regarding the composite resin restoration after different periods of time after MTA filling for vital pulp therapy. To assess the influence of potential prognostic factors on the success rate of direct pulp capping, a univariate analysis was previously applied for a MTA group and $\mathrm{Ca}(\mathrm{OH})_{2}$ group. The following variables were discussed as possible influential factors: age, gender, tooth location, type of tooth, type of restoration, time span before placement of restoration, site of exposure, type of pulp exposure, size of restoration, quality of coronal restoration at follow-up, treatment providers, and recall time (20). No conspicuous factors could be identified in the MTA group. Three time periods; immediately, 1 day, and 7 days after MTA pulp capping were investigated in the present study.

The proper timing for composite resin restoration over MTA placement has not been studied extensively because etching, rinsing, and priming all affect MTA setting. The manufacturer's instructions about how to set MTA 
indicate that a cotton pellet should be placed over the setting MTA for four hours before placement of the final restoration. Hence, the patient has to be recalled for a second visit for the final restoration. Clinical data showed that the prognosis after direct pulp capping using MTA does not depend on the timing of final restoration ( $<2$ days or $\geq 2$ days) (20). In another study, the shear bond strength (SBS) between MTA and composite resin was measured 48 hours after MTA mixing (21). An appropriate SBS (approximately 13 MPa) was obtained using an adhesive agent. However, the surface structural changes of MTA after composite resin restoration after various time intervals, including a short time lapse, have not yet been examined with regard to the physical properties. The aim of this study was to investigate the proper timing of restoring composite resin over MTA. The test hypothesis was that a very early composite resin restoration over MTA is possible under conditions that are sufficient for moist curing.

\section{Materials and Methods}

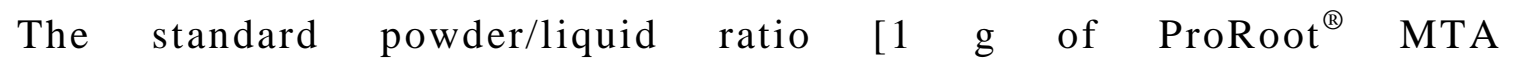
(DENTSPLY/Tulsa Dental, Tulsa, OK) powder to $0.33 \mathrm{~mL}$ of purified water] recommended in the manufacturer's instructions was used for this study. The mixed MTA was placed at a $3 \mathrm{~mm}$ in thickness in silicone tubes (5 $\mathrm{mm}$ high $\times 4 \mathrm{~mm}$ in diameter). A total of 35 samples of MTA blocks were divided into seven groups (each group: 5 samples) for the analyses. 


\section{Preparation of Samples}

For the control group, MTA was added to the tubes after mixing for 1 minute, and a moist cotton pellet was placed on the MTA. A 2 mm layer of hydraulic temporary cement $\left(\operatorname{Lumicon}^{\circledR}\right)$ (Heraeus-Kulzer Co, Germany) was placed on top of the MTA, and the MTA was maintained in the container for 21 days at room temperature and a relative humidity of $100 \%$.

For the 10-minute group without bonding agent, 10 minutes after MTA mixing, 2 mm of flowable composite resin (Clerfil ${ }^{\circledR}$ Majesty ${ }^{\circledR}$ LV, Shade: A3) (Kuraray Medical Co, Tokyo) was added on top of the MTA, and was light-cured for $60 \mathrm{~s}$ with a LED curing light source (PENCURE; Morita Co, Kyoto, Japan). The tube was maintained for 21 days at room temperature at a relative humidity of $100 \%$.

In the 10-minute group with bonding agent, 10 minutes after MTA mixing, the Clearfil ${ }^{\circledR}$ Megabond $^{\circledR}$ primer (Kuraray Medical Co, Tokyo) and the Clearfil $^{\circledast}$ Megabond $^{\circledast}$ bonding agent (Kuraray Medical Co, Tokyo) were applied on the MTA according to the manufacturer's instructions. Thereafter, the same procedures as done in the 10 -minute group without bonding agent were carried out for composite resin restoration and MTA maintenance.

For the 1-day group without bonding agent, a moist cotton pellet was placed on the MTA after it was added to the tube. Lumicon ${ }^{\otimes}$ was placed at a 2 mm in thickness on MTA, and MTA was maintained at room temperature and $100 \%$ relative humidity. One day after MTA filling, the Lumicon $^{\circledR}$ and a

moist cotton pellet with small fragments of Lumicon ${ }^{\circledR}$ were removed, and the 
MTA surface was air-blown. Next, the same procedures performed for 10-minute group without bonding agent were carried out for composite resin restoration and MTA maintenance.

In the 1-day group with bonding agent, samples were treated as in the 1-day group without bonding agent, but both a primer and bonding agent were used, as done in the 10-minute group with bonding agent.

For the 7-day group without bonding agent, after filling the tube with MTA, a moist cotton pellet was placed on the MTA. Lumicon ${ }^{\circledR}$ was placed at a $2 \mathrm{~mm}$ in thickness on the MTA, and the tube was maintained at room temperature and $100 \%$ relative humidity. Seven days after MTA filling, the MTA surface was air-blown as in the 1-day group, and composite resin restoration and MTA maintenance were done as in the 10-minute group without bonding agent.

In the 7-day group with bonding agent, samples were treated as in the 7-day group without bonding group, but both a primer and bonding agent were used, as done in the 10-minute group with bonding agent.

Twenty-one days after MTA mixing, all samples were removed from the silicone tubes, were embedded in epoxy resin (SpeciFix-20; Streus A/S, Ballerup, Denmark), and were maintained in a container for one week at room temperature. Thereafter, they were cut longitudinally into halves with an IsoMet $^{\circledR}$ (Buehler, Lake Bluff, IL). The longitudinal planes were then ground with silicon carbide papers up to $\# 1500$ grit, and finally polished with Diamond Abrasive Films (South Bay technology, Inc, San Clemente, CA) up to $0.5 \mu \mathrm{m}$ in size. 


\section{Scanning Electron Microscopy (SEM)}

The samples were mounted on aluminum holders with adhesive carbon tape and observed using an operating microscope. Thereafter, the same samples were coated with carbon using a vacuum evaporator (JEE-400; JEOL Ltd, Tokyo, Japan). They were then examined with a scanning electron microscope (S-3500N; Hitachi Ltd, Tokyo, Japan). SEM (magnification: $\times$ $500, \times 2,000)$ was conducted using a secondary electron imaging technique, with instrument operating at $15 \mathrm{kV}$ and a working distance of $15 \mathrm{~mm}$. The distances between the MTA and composite resin, or between the MTA and bonding agent on longitudinal planes were measured at five points at $0.5 \mathrm{~mm}$ intervals at the interface (magnification: $\times 2,000$ ).

\section{Vickers Microhardness Measurements}

The microhardness measurements were performed with a Vickers microhardness tester (Akashi MVK-H-M; Mitsutoyo Co, Kawasaki, Japan). The microhardness was measured on the MTA surface at five points at 0.5 $\mathrm{mm}$ intervals at a $200 \mu \mathrm{m}$ of distance from the interface using a Vickers indenter at a $50 \mathrm{gf}$ of load and with a $5 \mathrm{~s}$ dwell time. The angle between the opposite faces of the diamond indenter was $136^{\circ}$. The mean microhardness value of each specimen was calculated, and the values were expressed as the means \pm standard deviations (SD).

\section{Statistical Analyses}


The obtained Vickers microhardness data were evaluated statistically using the StatView software (version 5.0). The difference between the groups with and without bonding resin for each time interval before composite resin restoration was compared using an unpaired Student's t-test. The differences based on the time intervals before composite resin restoration in each group without or with bonding agent were analyzed by a one-way ANOVA, and multiple comparisons were evaluated by the Newman-Keuls test. Significance was set at the $5 \%$ level.

\section{Results}

\section{SEM}

In the 10-minute groups without and with bonding agent, no gap was observed between the MTA and composite resin, or between the MTA and bonding agent (Figs. $1 a$ and $b$ ). High magnification images (magnification: $\times 2.000$ ) of epoxy resin and bonding agent presented clear and different texture of surfaces each other. The former surface was observed as a scaly and not smooth appearance, but the latter as a smooth and flat appearance. Furthermore, the interface between composite resin and bonding agent was relatively smooth compared to that between composite resin and gap. Although complete separation at the interface between the MTA and composite resin occurred in all specimens in the 1-day group without bonding agent (Fig. 1c), no gap was observed between the MTA and bonding

agent in the 1-day group with bonding agent (Fig. 1d). In the 7-day group 
without bonding agent, some parts of the interface were in contact between the MTA and composite resin, however, the gap (from 4 to $40 \mu \mathrm{m}$ in length) occurred at other parts of the interface between the MTA and composite resin (Fig. 1e). In the 7-day group with bonding agent, no gap was observed between the MTA and bonding agent (Fig. 1f).

\section{Vickers Microhardness}

Table 1 shows the microhardness values in the seven groups. There were no significant differences between the composite resin restoration with and without the bonding agent at all time points examined $(P>0.05)$. ANOVA showed that the application time for composite resin restoration affected the microhardness $(P<0.01)$, regardless of the presence or absence of a bonding agent. In the groups treated without a bonding agent, except between the 10 -minute and control groups $(P>0.05)$, there were significant differences between groups $(P<0.05)$. In the groups treated with a bonding agent, a significant difference was observed between the 10-minute and 1-day groups, between the 1-day and 7-day groups, and between the 1-day and control groups $(P<0.01)$. However, there were no significant differences between the 10- minute and 7-day groups, between the 10-minute and control groups, and between the 7-day and control groups $(P>0.05)$. Totally, the 10 -minute groups presented the higher values similarly as the control group.

\section{Discussion}


The main chemical compositions are similar between MTA and Portland cement, although the quantitative results of trace elements exhibits marked differences (22). Therefore, the basic setting behavior of MTA is thought to be similar to that of Portland cement. The most important factor affecting Portland cement setting is curing (23). The mean setting time of MTA was $165 \pm 5$ minutes, and at 24 hours, MTA had the lowest compressive strength (40 MPa), but this increased to $67 \mathrm{MPa}$ after 21 days (19).

A spectral analysis previously demonstrated that the placement of glass ionomer cement (GIC) over MTA after 45 minutes did not affect its setting reaction, and that calcium salts may be formed in the interface of these two materials (24). It could therefore be concluded that conventional GIC might be layered over partially set MTA after 45 minutes and could be used for single visit procedures (25). The microhardness of MTA is affected by many factors, such as the $\mathrm{pH}(26,27)$, humidity and setting time (28), thickness of the material (29), condensation pressure (30), the acid-etching procedure (31), the setting conditions (32), EDTA treatment (33). The microhardness value also significantly increased after SEM \{personal communication, the value before SEM: $67.09+26.01(n=5)\}$. In the clinical setting, further uncontrolled factors such as bleeding and exudation from pulp related to individual cases are also present. In the 10-minute groups in the present study, SEM revealed interlocking areas at the interface due to roughness of MTA, and the microhardness of the MTA at the interface showed a high value similar to the control. The initial setting of the MTA has not yet been completed at this time. In the 10-minute group treated using a bonding agent, 
the self-etching procedure for composite resin restoration did not disturb the microhardness of the MTA, so the moist curing for MTA may be sufficient during this procedure. Therefore, the early covering of MTA with composite resin allows for sufficient moist curing, and does not prevent the setting reaction of the MTA.

In the 1-day groups, the microhardness decreased remarkably regardless of the presence or absence of a bonding agent. The dehydration of MTA surface due to air-drying before composite resin filling resulted in the prevention of moist curing and a significant decrease in the microhardness value. This negative effect of air-blowing on MTA surface was also recognized even in the 7 -day groups treated with the bonding agent. These findings suggest that when MTA is mixed as per the manufacturer's instructions, a composite resin could be successfully placed on the MTA, similarly to GIC restoration, without affecting its hardness.

In conclusion, when hydration during the MTA setting process is guaranteed, there are no particular problems related to composite resin restoration even if it is performed almost immediately after MTA filling as a recommended clinical procedure.

\section{References}

1. Camilleri J, Montesin FE, Brady K, et al. The constitution of mineral trioxide aggregate. Dent Mater 2005;21:297-303. 
2. Torabinejad M, Watson TF, Pitt Ford TR. Sealing ability of a mineral trioxide aggregate when used as a root end filling material. J Endod 1993;19:591-5.

3. Torabinejad M, Higa RK, McKendry DJ, et al. Dye leakage of four root end filling materials: effects of blood contamination. J Endod 1994;20:159-63.

4. Aminoshariae A, Hartwell GR, Moon PC. Placement of mineral trioxide aggregate using two different techniques. J Endod 2003;29:679-82.

5. Vizgirda PJ, Liewehr FR, Patton WR, et al. A comparison of laterally condensed gutta-percha, thermoplasticized gutta-percha, and mineral trioxide aggregate as root canal filling materials. J Endod 2004;30:103-6.

6. Yeung P, Liewehr FR, Moon PC. A quantitative comparison of the fill density of MTA produced by two placement techniques. J Endod 2006;32:456-9.

7. Bogen G, Kuttler S. Mineral trioxide aggregate obturation: a review and case series. J Endod 2009;35:777-90.

8. Lee SJ, Monsef M, Torabinejad M. Sealing ability of a mineral trioxide aggregate for repair of lateral root perforation. J Endod 1993;19:541-4.

9. Ford TR, Torabinejad M, McKendry DJ, et al. Use of mineral trioxide aggregate for repair of furcal perforation. Oral Surg Oral Med Oral Pathol Oral Radiol Endod 1995;79:756-63.

10. Ford TR, Torabinejad M, Abedi HR, et al. Using mineral trioxide aggregate as a pulp-capping material. J Am Dent Assoc 1996;127:1491-4.

11. Faraco IM Jr, Holland R. Response of the pulp of dogs to capping with 
mineral trioxide aggregate or a calcium hydroxide cement. Dental Traumatol 2001;17:163-6.

12. Aeinehchi M, Eslami B, Ghanbariha M, et al. Mineral trioxide aggregate (MTA) and calcium hydroxide as pulp-capping agents in human teeth: a preliminary report. Int Endod J 2003;36:225-31.

13. Nair PN, Duncan HF, Pitt Ford TR, et al. Histological, ultrastructural and quantitative investigations on the response of healthy human pulps to experimental pulp capping with mineral trioxide aggregate: a randomized controlled trial. Int Endod J 2008;41:128-50.

14. Tabarsi B, Parirokh M, Eghbal MJ, et al. A comparative study of dental pulp response to several pulpotomy agents. Int Endod J 2010;43:565-71.

15. Witherspoon DE, Ham K. One-visit apexification: technique for inducing root-end barrier formation in apical closures. Pract Proced Aesthet Dent 2001;13:455-60; quiz 462.

16. Hachmeister DR, Schindler WG, Walker WA 3rd, et al. The sealing ability and retention characteristics of mineral trioxide aggregate in a model of apexification. J Endod 2002;28:386-90.

17. Eidelman E, Holan G, Fuks AB. Mineral trioxide aggregate vs formocresol in pulpotomized primary molars: a preliminary report. Pediatr Dent 2001;23:15-8.

18. Holland R, de Souza V, Murata SS, et al. Healing process of dog dental pulp after pulpotomy and pulp covering with mineral trioxide aggregate or Portland cement. Braz Dent J 2001;12:109-13.

19. Torabinejad M, Hong CU, McDonald F, et al. Physical and chemical 
properties of a new root-end filling material. J Endod 1995;21:349-53.

20. Mente J, Geletneky B, Ohle $\mathrm{M}$, et al. Mineral trioxide aggregate or calcium hydroxide direct pulp capping: an analysis of the clinical treatment outcome. J Endod 2010;36:806-13.

21. Tunc ES, Sonmez IS, Bayrak S, et al. The evaluation of bond strength of a composite and a compomer to white mineral trioxide aggregate with two different bonding systems. J Endod 2008;34:603-5.

22. Dammaschke T, Gerth HU, Züchner H, et al. Chemical and physical surface and bulk material characterization of white ProRoot MTA and two Portland cements. Dent Mater 2005;21:731-8.

23. The Standard Specifications for Concrete Structures. Chapter 8 Curing pp. 176-180, 2007; The Japan Society of Civil Engineers' Concrete Committee.

24. Nandini S, Ballal S, Kandaswamy D. Influence of glass-ionomer cement on the interface and setting reaction of mineral trioxide aggregate when used as a furcal repair material using laser Raman spectroscopic analysis. J Endod 2007;33:167-72.

25. Ballal S, Venkateshhahu N, Nandini S, et al. An in vitro study to assess the setting and surface crazing of conventional glass ionomer cement when layered over partially set mineral trioxide aggregate. J Endod 2008;34:478-80.

26. Walker MP, Diliberto A, Lee C. Effect of setting conditions on mineral trioxide aggregate flexural strength. J Endod 2006;32:334-6.

27. Namazikhah MS, Nekoofar MH, Sheykhrezae MS, et al. The effect of pH 
on surface hardness and microstructure of mineral trioxide aggregate. Int Endod J 2008;41:108-16.

28. Gancedo-Caravia L, Garcia-Barbero E. Influence of humidity and setting time on the push-out strength of mineral trioxide aggregate obturations. J Endod 2006;32:894-6.

29. Matt GD, Thorpe JR, Strother JM, et al. Comparative study of white and gray mineral trioxide aggregate (MTA) simulating a one- or two-step apical barrier technique. J Endod 2004;30:876-9.

30. Nekoofar MH, Adusei G, Sheykhrezae MS, et al. The effect of condensation pressure on selected physical properties of mineral trioxide aggregate. Int Endod J 2007;40:453-61.

31. Kayahan MB, Nekoofar MH, Kazandag M, et al. Effect of acid-etching procedure on selected physical properties of mineral trioxide aggregate. Int Endod J 2009;42:1004-14.

32. Walker MP, Diliberto A, Lee C. Effect of setting conditions on mineral trioxide aggregate flexural strength. J Endod 2006;32:334-6.

33. Lee Y-L, Lin F-H, Wang W-H, et al. Effects of EDTA on the hydration mechanism of mineral trioxide aggregate. J Dent Res 2007;86:534-8. 


\section{Figure Legend}

Figure 1. A scanning electron micrograph of the interface between the MTA and composite resin (CR), or between the MTA and bonding agent (MB) (original magnification, $\times 500)$. (a) The 10-minute group without bonding agent. (b) The 10-minute group with bonding agent. (c) The 1-day group without bonding agent. Note the artificial space $(S) .(d)$ The 1-day group with bonding agent. (e) The 7-day group without bonding agent. Note the gap formation (G). ( $f$ ) The 7-day group with bonding agent. 
Figure 1.
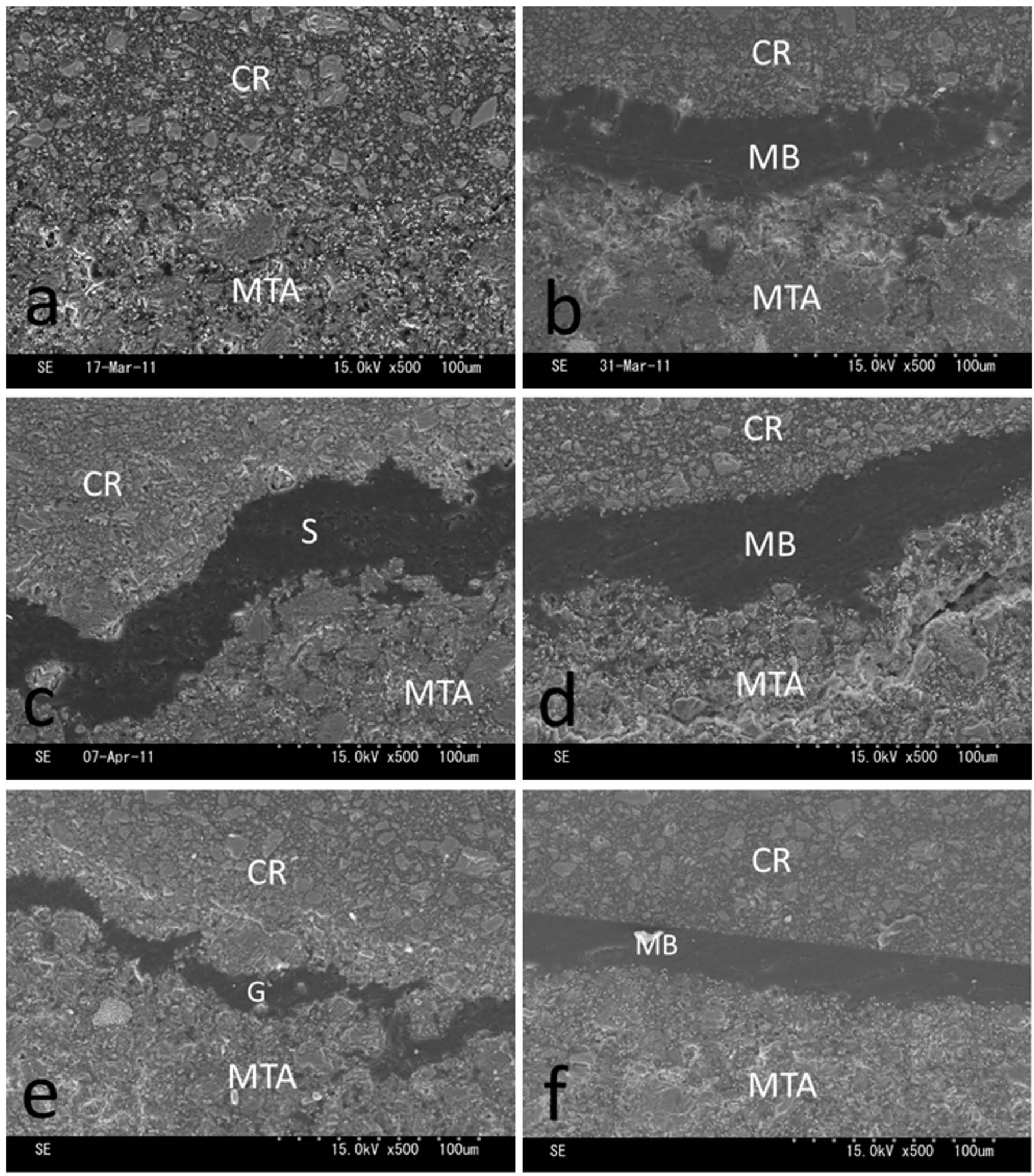
TABLE 1. Mean microhardness of MTA and SD at various times

\begin{tabular}{lcccc}
\hline Bonding agent & 10 minutes & 1 day & 7 days & Control \\
\hline Absence & $111.37 \pm 13.95$ & $70.44 \pm 16.18$ & $85.33 \pm 28.86$ & $106.17 \pm 26.00$ \\
Presence & $102.45 \pm 18.23$ & $68.26 \pm 19.22$ & $94.50 \pm 30.02$ & \\
\hline
\end{tabular}

